\title{
Long-term treatment with alendronate increases the surgical difficulty during simple exodontias - an in vivo observation in Holtzman rats
}

\author{
Nicolau Conte-Neto ${ }^{1}$, Alliny de Souza Bastos ${ }^{1+}$, Luis Carlos Spolidorio ${ }^{2 \dagger}$, \\ Rosemary Adriana Chierici Marcantonio ${ }^{1+}$ and Elcio Marcantonio $\mathrm{Jr}^{1 *}$
}

\begin{abstract}
Background: Atraumatic teeth extractions protocols are highly encouraged in patients taking bisphosphonates (Bps) to reduce surgical trauma and, consequently, the risk of jaws osteonecrosis development. In this way, this paper aims to report the findings of increased surgical difficulty during simple exodontias in animals treated with bisphosphonates.

Methods: Sixty male Holtzman rats were randomly distributed into three groups of 20 animals and received daily subcutaneous administration of $1 \mathrm{mg} / \mathrm{kg}$ (AL1) or $3 \mathrm{mg} / \mathrm{kg}$ (AL3) of alendronate or saline solution (CTL). After 60 days of drug therapy all animals were submitted to first lower molars extractions under general anesthesia. Operatory surgical time and the frequency of teeth fractures were measured as principal outcomes and indicators of surgical difficulty degree.
\end{abstract}

Results: Animals treated with alendronate (AL1 and AL3) were associated to higher operatory times and increased frequency of teeth fractures compared to match controls.

Conclusions: The bisphosphonate therapy may be associated with an increased surgical difficulty and trauma following simple exodontias protocols, which is considered a critical issue when it comes to osteonecrosis development.

Keywords: Bisphosphonates, Tooth extraction, Osteonecrosis

\section{Background}

Tooth Extraction is one of the most common procedures in oral surgery practice and the difficulty to perform this procedure varies according to a sort of risk factors. Among these factors, the increased bone density has been recognized as a relevant feature $[1,2]$ that can be an aging physiologic issue or be resulted of antiresorptive drugs, including bisphosphonates (Bps) [3].

Bps have been widely used to stabilize bone loss occasioned by bone disorders, such as osteoporosis and Paget disease (Rogers et al. 2000). In this way, it has been generating a great concern due to the increasing number of

\footnotetext{
* Correspondence: elciojr@foar.unesp.br

${ }^{\dagger}$ Equal contributors

'Department of Diagnosis and Surgery, Division of Periodontology, UNESPUniv. Estadual Paulista, School of Dentistry, Rua Humaitá, 1680, Araraquara, SP 14801-903, Brazil

Full list of author information is available at the end of the article
}

Bisphosphonate-related Osteonecrosis of the Jaws (BRONJ). Considering the strong correlation between the etiology of this bone disease with tooth extractions [4-6] many efforts, have been made to reduce the surgical trauma in these patients $[7,8]$. In this context this paper aims to report the findings of increased surgical difficulty, based on the analysis of operatory time and teeth fractures frequency, associated with bisphosphonate therapy.

\section{Material and methods \\ Animals}

Sixty male Holtzman rats weighting 155 to $200 \mathrm{~g}$ were used and randomly distributed into three groups of 20 animals each. The rats were housed in polypropylene cages in groups of five animals per cage, at controlled room temperature $\left(23 \pm 20^{\circ} \mathrm{C}\right)$, humidity $(55 \pm 10 \%)$, and

\section{Biomed Central}

(c) 2012 Conte-Neto et al.; licensee BioMed Central Ltd. This is an Open Access article distributed under the terms of the Creative Commons Attribution License (http://creativecommons.org/licenses/by/2.0), which permits unrestricted use, distribution, and reproduction in any medium, provided the original work is properly cited. 
12/12 h light/dark cycle beginning at 7:00 a.m. Standard chow and tap water were available ad libitum. All the protocols described here were approved by local Ethics Committe of the School of Dentistry of Araraquara, São Paulo, Brazil (Protocol number 18/2009).

\section{Treatments}

The animals received daily subcutaneous doses of alendronate (Ale, 1 or 3 mg/kg; ALCON, São Paulo, Brazil), or saline solution $(0.9 \% \mathrm{NaCl}$; control group). After 60 days of alendronate or saline solution treatment, all animals were submitted to lower first molars extractions under general anesthesia using an intraperitoneal injection of ketamine $(0.1 \mathrm{ml} / 100 \mathrm{~g}$ body weight).

\section{Teeth extraction}

Teeth extractions were performed by the same operator with the same technique in all animals. Initially, the rats were placed in a dorsal position and fixed in a special device. After that, the surrounding gingival was carefully detached from the lower first molars with a dental explorer and teeth luxation were made using a Hollenback Carver followed by the tooth removal with a forceps, adapted around the cervical line of the tooth.

\section{Difficulty factors risk indicators}

The assessment of extraction difficulty degree was based on teeth fractures frequency and on the time spent to perform the extraction. This last parameter was defined as the interval between the utilization of the first instrument required to the tooth extraction until the use of the last instrument. Extraction time was measured using a digital timepiece for each case included in the study. The same individual (A.S.B.) measured all extraction times [9] to reduce possible bias. Furthermore, both individuals (operator and A.S.B.) were blinded to the study groups' assessment.

Teeth fractures frequency were defined as a complete loose in tooth continuity involving crown and/or roots [10].

\section{Statistical analysis}

The data were evaluated using the GraphPad Prism 5.0 software package (GraphPad Inc., San Diego, CA USA). The normality of the data was assessed by the Kolmogorov-Smirnov test. Comparisons among groups were performed using the chi-squared test to teeth fractures frequency analysis and Kruskal-Wallis followed by Dunns post test to surgery duration evaluation (nonparametric data). Results are presented as frequency of teeth fractures. Statistical significance was set at 5\% with 95\% confidence intervals.

\section{Results}

During lower first molars extractions of experimental animals, surgical difficulty was markedly increased in animals treated with alendronate $1 \mathrm{mg}\left(\mathrm{AL}_{1}\right)$ and $3 \mathrm{mg}$ $\left(\mathrm{AL}_{3}\right)$ when compared to control group (CTL). Group $\mathrm{AL}_{1}$ and $\mathrm{AL}_{3}$ animals presented a higher operatory time when compared to animals in control group (Figure 1).

Moreover, in the teeth fractures frequency analysis it was observed a significant difference between the groups. While animals in CTL group presented $8 \%$ of teeth fractures, animals in alendronate group presented $39.4 \%$ $\left(\mathrm{AL}_{1}\right)(\mathrm{p}<0.01)$ and $62.5 \%\left(\mathrm{AL}_{3}\right)(\mathrm{p}<0.001)$. Thus, comparing $\mathrm{AL}_{1}$ with $\mathrm{AL}_{3}$ groups, it was observed that $\mathrm{AL}_{3}$ animals presented teeth fractures frequency significantly increased compared with $\mathrm{AL}_{1}$ group $(\mathrm{p}<0.05)$ (Figure 2).

\section{Discussion}

In fact, the assessment of surgical difficulty is a relevant issue in the field of oral surgery since allows health professionals to plan operations more accurately helping to minimize surgical trauma, and risks of accidents and complications. This concern is highly relevant to patients treated with bisphosphonates due to the jaws osteonecrosis risk.

The assessment of extraction difficulty has been measured via a wide range of variables [9] and, among of them, the extraction time $[11,12]$ and complications frequency $[13,14]$ are well recognized indicators. For this reason, we selected these two variables as the outcome measures to evaluate the relationship of bisphosphonate therapy with surgical difficulty.

Our findings demonstrated that teeth extractions in animals treated with bisphosphonate require more surgical time when compared to control animals following simple extraction technique. In our opinion, the main

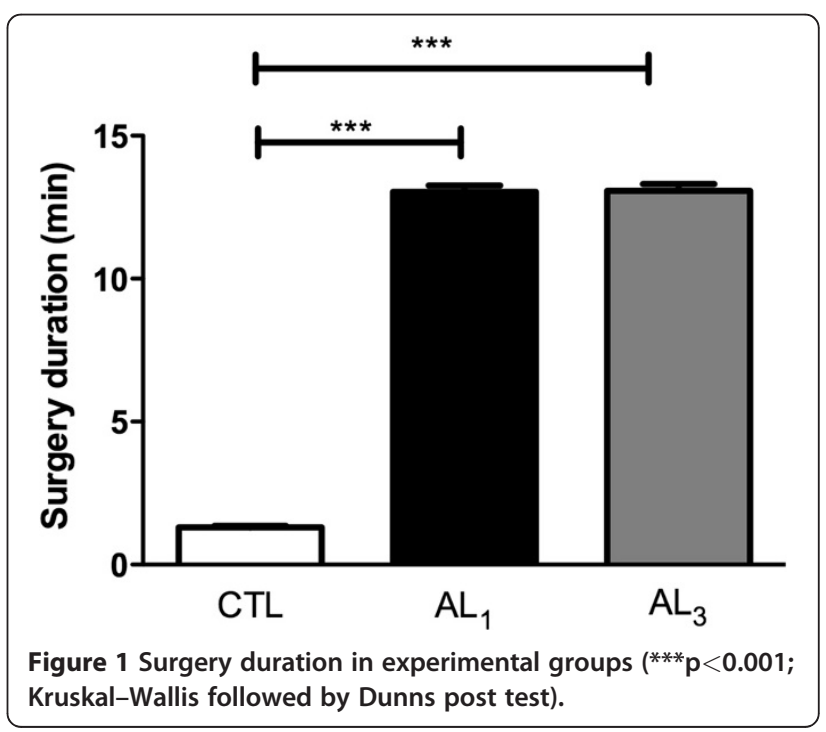




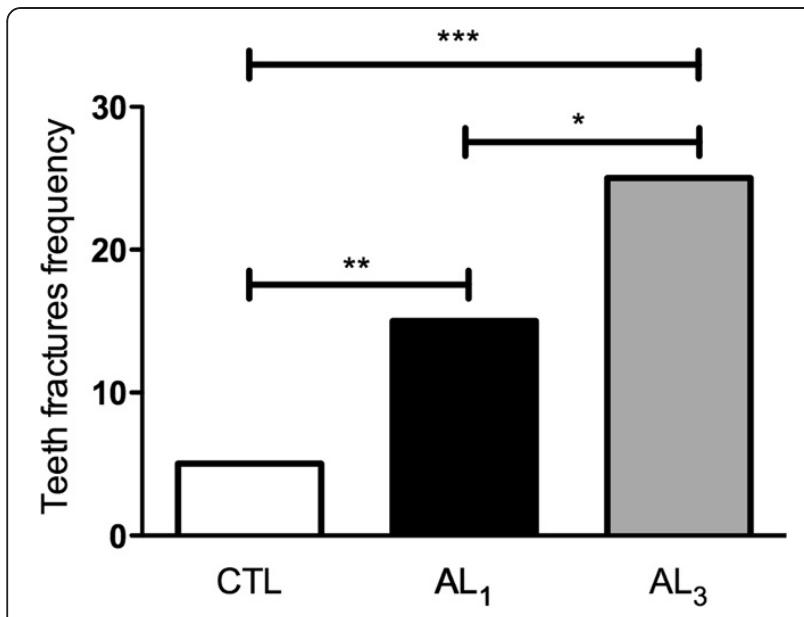

Figure 2 Teeth fractures frequency in experimental groups. A total of 40 teeth extractions were performed for each group $\left({ }^{*} p<0.05 ;{ }^{* *} p<0.01 ;{ }^{* * *} p<0.001\right.$; chi-squared test).

reason to justify this surgical time difference is related to the presence of an increased bone density and consequently decrease bone elasticity, that are well recognized difficult factors to teeth extractions $[1,2]$ and are a result of Bps treatment [3].

Even with the absence of bone density evaluation and measurement methods, it is reasonably to believe that Bps treated animals presented a high bone density that is supported by the following points:

1- It is known that the bone effect of Bps is cumulative and assumes a bone absorption linear aspect until $5 \mathrm{mg} / \mathrm{kg}$ endovenously dosages [15]. Therefore, the long-term treatment of Bps in high dosages (1 and $3 \mathrm{mg} / \mathrm{kg}$ ) used in this study resulted in an expressive Bps bone effect.

2- Bps were administered by subcutaneous route that are as effective as endovenously route regarding to drug bioavailability [15]. By this route it is estimated that more of $50 \%$ of the drug is available for bone matrix incorporation [16,17].

3- Due to the high bone turnover in cortical alveolar bone is believed that, although controversial, the Bps bone absorption is higher when compared to other skeletal sites [18], which can be justify by the alveolar lamina dura sclerosis seen in Bps treated patients with BRONJ in initial stage [19]. Besides, the mandibular bone has by itself a higher tissue degree of mineralization when compared to maxillae, been more prone to Bps effects and naturally increases surgical difficulty [9].

In this way, when it is opted to a simple exodontias technique, there is a highly dependence of the tooth to expand the bone tooth socket walls to allow its avulsion and in situations of an alveolar bone increased density there is a lack of sufficient socket expansion which obviously limits the teeth avulsion axis. Consequently, as it happened in this study, requires more surgical manipulation, thereby prolonging operating time [9], as well as the surgical trauma and increases the risk of accidents and complications.

Teeth fractures have been considerate the most frequent accidents during exodontias, in oral surgery practice [20]. They are usually related to inadequate instrumental use and excessive force use, which was one of the reasons that could justify the high frequency of teeth fractures in animals treated with Bps observed in this paper. The concern about this issue is that many efforts have been made to reduce the surgical trauma during teeth extractions by using atraumatic protocols in Bps treated patients $[7,8]$ since exodontias have been considerate as one the most frequent trigger factor to BRONJ [4-6].

In this context, as stated previously, in situations of an increased surgical difficulty degree there is a tendency to prolong surgical length and increase tissue trauma which in field of ONJ can lead to relevant implications:

1- Increase the inflammation of the alveolar bone [21], which could act in favor of the BRONJ lesions development according to the inflammatory theory [22].

2- Result in delayed extraction wound healing due to the compression of bone lining the socket impairing vascular penetration and results in thrombosis of the vessels [21], which could act in favor of the BRONJ lesions development according to the angiogenic theory [23].

3- Increase the risk of dento-alveolar fractures, since when bone tissue becomes too highly mineralized, it also becomes brittle [24]. Moreover, it also makes the tissue more prone to microcrack initiation [25], which act in favor to the BRONJ lesions development according to the bone suppression theory [26].

Another concern that can be discussed regarding to the teeth fractures is about the approaches after these accidents:

1- If opted to extract the residual fragment, the surgical time can be prolonged and increases the tissue trauma, being sometimes necessary to perform bone removal techniques, which can contribute to BRONJ lesions as stated previously.

2- If opted to keep the residual fragment and follow the patient, the surgical trauma will be obviously lower; however, eventually tooth or bone fragments/ remnants can lead to an increase in the risk of 
socket infection [21], which could also increase the risk of osteonecrosis according to the infectious theory [26].

Considering that the more atraumatic is the teeth extraction the better is for the healing process, with special mention in Bps treated patients, we highlight the strategies that can reduce the force intensity and the risk of teeth fracture during the exodontias, such as odontotomy techniques. Extractions without tooth sectioning might be responsible for a more traumatic and difficult surgery, especially in light of difficulty factors, such as increased bone density that can lead to several complications related to BRONJ lesions.

\section{Conclusions}

The bisphosphonate therapy may be associated with an increased surgical difficulty and trauma following simple exodontias protocols, which is considered a critical issue when it comes to osteonecrosis development.

\section{Consent}

A copy of the written consent form is available for review by the Editor-in-Chief of this journal.

\section{Competing interests}

The authors declare that they have no competing interests.

\section{Authors' contributions}

NCN performed the surgical procedures. ASB helped with the

methodological parameters. LCS, RACM and EMJ drafted the manuscript and reviewed it critically. All authors read and approved the final version of the manuscript.

\section{Authors' information}

NCN is a PhD student from Implantology program at Araraquara School of Dentistry and ASB is a PhD student from Periodontology program at Araraquara School of Dentistry. LCS is a professor and the chairman of the Department of Physiology and Pathology, Division of Pathology at Araraquara School of Dentistry. EMJ and RACM are professors and chairmen of the Department of Diagnosis and Surgery, Division of Periodontology at Araraquara School of Dentistry.

\section{Author details}

'Department of Diagnosis and Surgery, Division of Periodontology, UNESPUniv. Estadual Paulista, School of Dentistry, Rua Humaitá, 1680, Araraquara, SP 14801-903, Brazil. School of Dentistry, Department of Physiology and Pathology, Division of Pathology, UNESP - Univ. Estadual Paulista, Rua Humaitá, 1680, Araraquara, SP 14801-903, Brazil.

Received: 12 April 2012 Accepted: 13 July 2012

Published: 26 July 2012

\section{References}

1. Bruce RA, Frederickson GC, Small GS: Age of patients and morbidity associated with mandibular third molar surgery. J Am Dent Assoc 1980, 101:240-245.

2. Malkawi Z, Al-Omiri MK, Khraisat A: Risk indicators of postoperative complications following surgical extraction of lower third molars. Med Princ Pract 2011, 20:321-325.

3. Richer E, Lewis MA, Odvina CV, Vazquez MA, Smith BJ, Peterson RD, et al: Reduction in normalized bone elasticity following long-term bisphosphonate treatment as measured by ultrasound critical angle reflectometry. Osteoporos Int 2005, 16:1384-1392.
4. Thumbigere-Math V, Sabino MC, Gopalakrishnan R, Huckabay S, Dudek AZ, Basu S, et al: Bisphosphonate-related osteonecrosis of the jaw: clinical features, risk factors, management, and treatment outcomes of 26 patients. J Oral Maxillofac Surg 2009, 67:1904-1913.

5. Treister N, Sheehy N, Bae EH, Friedland B, Lerman M, Woo S: Dental panoramic radiographic evaluation in bisphosphonate-associated osteonecrosis of the jaws. Oral Dis 2009, 15:88-92.

6. Vieillard MH, Maes JM, Penel G, Facon T, Magro L, Bonneterre J, et al: Thirteen cases of jaw osteonecrosis in patients on bisphosphonate therapy. Joint Bone Spine 2008, 75:34-40.

7. Ferlito S, Puzzo S, Liardo C: Preventive protocol for tooth extractions in patients treated with zoledronate: a case series. J Oral Maxillofac Surg 2011, 69:e1-e4.

8. Scoletta M, Arduino PG, Pol R, Arata V, Silvestri S, Chiecchio A, et al: Initial experience on the outcome of teeth extractions in intravenous bisphosphonate-treated patients: a cautionary report. J Oral Maxillofac Surg 2011, 69:456-462.

9. Susarla SM, Dodson TB: Risk factors for third molar extraction difficulty. J Oral Maxillofac Surg 2004, 62:1363-1371.

10. Yuan LT, Duan DM, Tan L, Wang XJ, Wu LA: Treatment for a complicated crown-root fracture with intentional replantation: a case report with a 3.5-year follow up. Dent Traumatol 2012, . Epub ahead of print.

11. Renton T, Smeeton N, McGurk M: Factors predictive of difficulty of mandibular third molar surgery. Br Dent J 2001, 190:607-610.

12. Santamaria J, Arteagoitia I: Radiologic variables of clinical significance in the extraction of impacted mandibular third molars. Oral Surg Oral Med Oral Pathol Oral Radiol Endod 1997, 84:469-473.

13. Blondeau F, Daniel NG: Extraction of impacted mandibular third molars: postoperative complications and their risk factors. J Can Dent Assoc 2007, 73:325.

14. Bouloux GF, Steed MB, Perciaccante VJ: Complications of third molar surgery. Oral Maxillofac Surg Clin North Am 2007, 19:117-128. vii.

15. Lin JH: Bisphosphonates: a review of their pharmacokinetic properties. Bone 1996, 18:75-85

16. Berenson JR, Rosen L, Vescio R, Lau HS, Woo M, Sioufi A, et al: Pharmacokinetics of pamidronate disodium in patients with cancer with normal or impaired renal function. J Clin Pharmacol 1997, 37:285-290.

17. Ezra A, Golomb G: Administration routes and delivery systems of bisphosphonates for the treatment of bone resorption. Adv Drug Deliv Rev 2000, 42:175-195.

18. Reid IR: Bisphosphonates. Skeletal Radiol 2007, 36:711-714.

19. Hutchinson M, O'Ryan F, Chavez V, Lathon PV, Sanchez G, Hatcher DC, et al: Radiographic findings in bisphosphonate-treated patients with stage 0 disease in the absence of bone exposure. J Oral Maxillofac Surg 2010, 68:2232-2240.

20. Adeyemo WL, Ladeinde AL, Ogunlewe MO: Influence of trans-operative complications on socket healing following dental extractions. J Contemp Dent Pract 2007, 8:52-59

21. Birn H: Fibrinolytic activity of alveolar bone in "dry socket". Acta Odontol scand 1972, 30:23-32.

22. Lesclous P, Abi Najm S, Carrel JP, Baroukh B, Lombardi T, Willi JP, et al: Bisphosphonate-associated osteonecrosis of the jaw: a key role of inflammation? Bone 2009, 45:843-852.

23. Marx RE, Sawatari Y, Fortin M, Broumand V: Bisphosphonate-induced exposed bone (osteonecrosis/osteopetrosis) of the jaws: risk factors, recognition, prevention, and treatment. J Oral Maxillofac Surg 2005, 63:1567-1575

24. Currey JD, Brear K, Zioupos P: The effects of ageing and changes in mineral content in degrading the toughness of human femora. J Biomech 1996, 29:257-260.

25. Burr DB, Miller L, Grynpas M, Li J, Boyde A, Mashiba T, et al: Tissue mineralization is increased following 1-year treatment with high doses of bisphosphonates in dogs. Bone 2003, 33:960-969.

26. Allen MR, Burr DB: The pathogenesis of bisphosphonate-related osteonecrosis of the jaw: so many hypotheses, so few data. J Oral Maxillofac Surg 2009, 67:61-701.

doi:10.1186/1746-160X-8-20

Cite this article as: Conte-Neto et al.: Long-term treatment with alendronate increases the surgical difficulty during simple exodontias an in vivo observation in Holtzman rats. Head \& Face Medicine 2012 8:20. 